\title{
Characteristics and Histopathological Grading of Malignant Spiculated Mass in regards to Histopathological Grading of Breast Cancer Based on The Nottingham Grading System
}

\author{
Andi Syarti ${ }^{1}$, Ulinta Pasaribu ${ }^{2}$, Dyah Fauziah ${ }^{3}$, Lies Mardiyana ${ }^{2}$, Tri Wulanhandarini ${ }^{2 *}$ \\ ${ }^{1}$ Faculty of Medicine, Universitas Airlangga, Surabaya, Indonesia \\ ${ }^{2}$ Department of Radiology, Universitas Airlangga - Dr. Soetomo General Hospital, Surabaya, Indonesia \\ ${ }^{3}$ Department of Anatomical Pathology, Universitas Airlangga - Dr. Soetomo General Hospital, Surabaya, Indonesia
}

\section{ART I CLE I N FO}

\section{Article history:}

Received 13 May 2020

Received in revised form 03 June

2020

Accepted 08 June 2020

Available online 30 June 2020

Keywords:

Malignant mass,

Mammographic spiculation,

The Nottingham Grading System.

*) Corresponding author:

femaleimaging19@gmail.com

\begin{abstract}
A B S T R A C T
Introduction: Spiculation in mammography is a typical finding for invading breast cancer and is an important criterion in diagnosis and in predicting prognostic and plays an important role in management. The purpose of this research is to determine the characteristics of malignant spiculating mass in mammography in regards to histopathological grading using The Nottingham Grading System.

Methods: Patients whom had spiculation in mammography was reviewed using medical record data by two breast imaging consultants and then combined with The Nottingham Grading System criteria obtained from histopathological examination results of core biopsy and surgery specimen. There was 29 cases that met the inclusion criteria.

Results: Of the 29 patients that met the inclusion, the spiculated masses grade I, II has mitotic index of $0-12$, grade III has mitotic index of 13-25. Histopathologic grade II, III has the most tubular formation of $<10 \%$, grade I has $10-75 \%$. Grade I, II has moderate nuclear pleomorphism, grade III has severe nuclear pleomorphism. Most were grade III (44.8\%), followed by grade II (37.9\%), and minimally grade I (17.2\%). Most patients are in stage 3 breast cancer.

Conclusion: Malignant spiculated mass with grade I, II has low proliferation index (mitotic index 0 -12), histopathological grade II, III had worse cellular differentiation (tubular formation $<10 \%$ ), histopathological grade II, III has moderate to severe nuclear pleomorphism.
\end{abstract}

\section{Introduction}

Spiculation in mammography is a typical finding for invading breast cancer and is an important criterion for diagnosis. ${ }^{1}$ The incidence of breast cancer in Indonesia is around 1,4 in every 100 people. $^{2}$ Previous studies showed that a spiculated mass in mammography is also able to predict the prognostic factors which play a very important role in disease management. . $^{3,4}$ One of the strongest prognostic factors is the tumor histopathological grading (according to The Union for International Cancer Control (UICC)). ${ }^{5}$ A study by Lamb concluded that grade I and II spiculated mass in mammography is significant (72\%) as compared to grade III $(24 \%){ }^{6}$ Spiculated mass was significantly related to survival rate of breast cancer patients due to a strong correlation between low histopathological grade mass and spiculated on mammography. ${ }^{7}$ The World Health Organization (WHO), American Joint Committee on Cancer (AJCC), European Union (UE), and Royal College of Pathologists (UK RCPath) recommend The Nottingham Grading System (NGS) for histopathological grading which is a modification of Scarff-Blomm-Richardson grading system. The NGS is a simple scoring system to assess the grade of a breast cancer and to rate how aggressive a tumour may behave. Thus, determining the grade is very important to help the clinicians to give the right treatment options for the patients. The grading is based on three morphological features: 1). Mitotic index, 2). Degree of tubular /

Biomolecular and Health Science Journal.

Available at https://e-journal.unair.ac.id/BHSJ ; DOI: 10.20473/bhsj.v3i1.19134 
glandular formation, and 3). Nuclear pleomorphism. ${ }^{8}$

\section{Methods}

\section{Subject}

This study was a retrospective observational, descriptive study conducted from January 2017 to December 2018 at Dr. Soetomo General Hospital, Surabaya. Using consecutive sampling method, mammographic data in medical records were reviewed by two breast imaging consultants. The inclusion criteria were: 1). Complete bilateral mammography in females, 2). A spiculated mass found in the first mammographic evaluation, 3). Patient had not undergone any neoadjuvant chemotherapy or surgery before mammography was performed (not a recurrent or residual mass), 4). Histopathologic examination performed after mammography, 5). Pathologic report of malignant mass including its histopathological grading. Exclusion criteria were: 1). Mammographic evaluation on contralateral breast, 2). More than one mass in ipsilateral breast, 3). Previous mammography results unavailable (if post-therapeutic evaluation was performed during the sampling period). From a total of 75 cases, 29 cases fulfilled the criteria. This study was conducted after receiving written consent from the patient regarding biopsy and all examinations and had been approved by the ethics committee of Dr. Soetomo General Hospital, Surabaya (ethical clearance number 0964/KEPK/II/2019).

\section{Imaging and Histopathological Grading}

All mammograms were created using GE Senoclaire GE Mammography System (Senographe Essential 54.20, GE Healthcare, USA). Histopathological grading was assessed from breast tissue samples using hematoxylin $\&$ eosin stain and Olympus microscope (Olympus
Corporation, Japan) with 40x field of view diameter equals to $4 \mathrm{~mm}$. Magnifications used were 40x, 100x, 200x, and 400x and evaluated by a pathology consultant.

\section{Data Analysis}

All data recorded were analysed and presented descriptively using cross tabulation.

\section{Results}

Of 75 cases of breast cancer, 46 cases were excluded because had more than one mass, had contralateral breast cancer and did not provide previous mammography result. Total 29 cases were included in this study. The youngest patient was 34 years old and oldest was 73 years old with the average age 45.7 years old. Most patients are in stage 3 breast cancer.

Table 1 shows that from 5 spiculated masses with histopathological grade I (low), the mitotic index of $0-11$ and $12-22$ were $3(60 \%)$ and $2(40 \%)$ respectively. In terms of tubular formation, 4 cases $(80 \%)$ were $10-75 \%$ and 1 case $(20 \%)$ was $>75 \% .3(60 \%)$ masses had intermediate nuclear pleomorphism while the remaining $2(40 \%)$ had low nuclear pleomorphism.

From 11 spiculated masses with histopathological grade II (intermediate), $7(63.6 \%)$ had mitotic index of $0-11,3$ $(27.3 \%)$ had mitotic index of $12-22$. In terms of tubular formation, 8 cases $(72.7 \%)$ were $<10 \%, 2$ (18.2\%) cases were $10-75 \%$, and 1 case $(9.1 \%)$ was $>75 \%$. 9 cases $(81.8 \%)$ masses had intermediate nuclear pleomorphism while the remaining 2 (18.2\%) had high nuclear pleomorphism.

From 13 spiculated masses with histopathological grade III (high), 8 cases $(61.5 \%)$ had mitotic index of 12-22 and 5 cases $(38.5 \%)$ had mitotic index of $>23$. All of these high grade masses had $<10 \%$ tubular formation. 12 cases $(92.3 \%)$ masses had high nuclear pleomorphism while only $1(7.7 \%)$ had intermediate nuclear pleomorphism.

Table 1 Malignant spiculated mass and the histopathological grading based on The Nottingham Grading System parameters.

\section{The Nottingham Grading} System criteria
Low histopathologic grade (grade I)

Malignant spiculated mass
Intermediate histopatho-
logic grade
(grade II)

\section{High histopathologic grade (grade III)}

\begin{tabular}{|c|c|c|c|}
\hline \multicolumn{4}{|l|}{ Mitotic index } \\
\hline Mitotic index 0-11 & $3(60 \%)$ & $7(63.6 \%)$ & $0(0 \%)$ \\
\hline \multicolumn{4}{|l|}{ Mitotic index } \\
\hline $12-22$ & $2(40 \%)$ & $3(27.3 \%)$ & $8(61.5 \%)$ \\
\hline Mitotic index $>23$ & $0(0 \%)$ & $1(9.1 \%)$ & $5(38.5 \%)$ \\
\hline \multicolumn{4}{|l|}{ Tubular formation } \\
\hline Tubular formation $>75 \%$ & $1(20 \%)$ & $1(9.1 \%)$ & $0(0 \%)$ \\
\hline Tubular formation $10-75 \%$ & $4(80 \%)$ & $2(18.2 \%)$ & $0(0 \%)$ \\
\hline Tubular formation $<10 \%$ & $0(0 \%)$ & $8(72.7 \%)$ & $13(100 \%)$ \\
\hline \multicolumn{4}{|l|}{ Nuclear pleomorphism } \\
\hline Low & $2(40 \%)$ & $0(0 \%)$ & $0(0 \%)$ \\
\hline Intermediate & $3(60 \%)$ & $9(81.8 \%)$ & $1(7.7 \%)$ \\
\hline High & $0(0 \%)$ & $2(18.2 \%)$ & $12(92.3 \%)$ \\
\hline
\end{tabular}


Table 2. Histopathological grade of malignant speculated mass based on The Nottingham Grading System

\begin{tabular}{lcc}
\hline \multicolumn{1}{c}{ Histopathological grade } & Spiculated mass frequency & Percentage (\%) \\
\hline Grade I & 5 & 17.2 \\
\hline Grade II & 11 & 37.9 \\
\hline Grade III & 13 & 44.8 \\
\hline Total & 29 & 100 \\
\hline
\end{tabular}

Table 3. Distribution of size of malignant spiculated mass on mammography based on histopathological grading.

\begin{tabular}{lccc}
\hline \multirow{2}{*}{ Histopathological grade } & $\mathbf{2}$ & Size (cm) & Total \\
\hline Grade I & $2(40 \%)$ & $3(60 \%)$ & $5(100 \%)$ \\
\hline Grade II & $3(27.3 \%)$ & $8(72.7 \%)$ & $11(100 \%)$ \\
\hline Grade III & $1(7.7 \%)$ & $12(92.3 \%)$ & $13(100 \%)$ \\
\hline
\end{tabular}
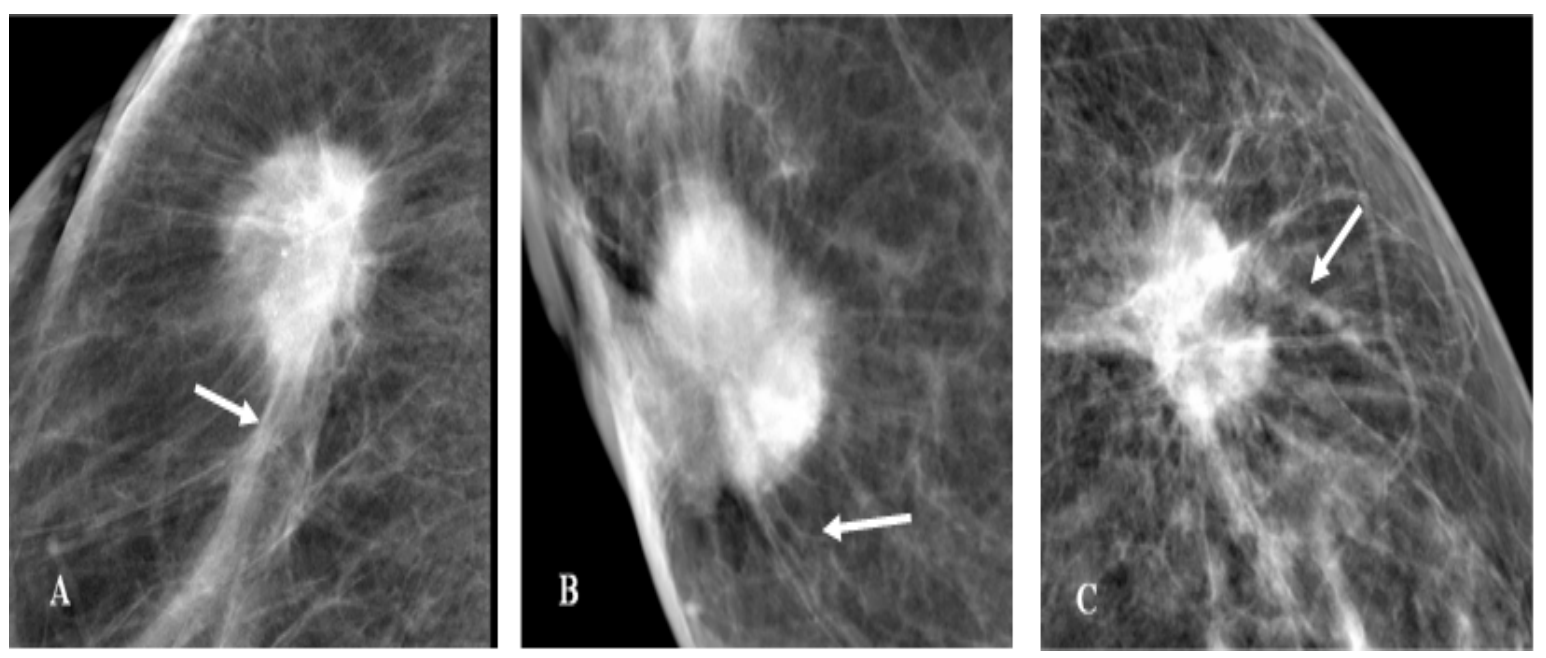

Figure 1. Spiculated masses with histopathological grade I (A), grade II (B), and grade III (C)

Table 2 shows that most of spiculated masses have histopathological grade III $(44.8 \%)$, followed by grade II $(37.9 \%)$ and grade I $(17.2 \%)$.

Based on histopathological grading, 2 cases $(40 \%)$ of grade I masses were $\leq 2 \mathrm{~cm}$ in size while the remaining 3 cases $(60 \%)$ were $>2 \mathrm{~cm}$. There were 8 cases $(72.7 \%)$ of grade II masses with size $>2 \mathrm{~cm}$. Around 12 cases $(92.3 \%)$ of grade III were $>2 \mathrm{~cm}$ (Table 3 ).

\section{Discussion}

Our study found spiculated masses were significantly seen more in high grade (grade III) than intermediate and low grade (grade II and grade I). On the other hand, previous study showed otherwise by demonstrating a statistically significant association between low and intermediate histopathological grades and the presence of spiculated mass. ${ }^{4,9}$

Previous study also showed an association between spiculated mass on mammography with luminal Amolecular subtype of invasive breast cancer compared to other molecular subtypes. Luminal A of breast cancer subtype has a very good response with endocrinotherapy and has a good prognostic factor. However, it can also read as a discrepancy in the examination if combine with hormone receptor and immunohistochemistry Thus, spiculated findings on mammography can be used as predictors that can assist clinicians in the selection of therapeutic management before histochemical examination. ${ }^{4}$

Most of the spiculated masses in this study were more than $2 \mathrm{~cm}$ in size, whereas most of them in previous studies are less than or equal to $2 \mathrm{~cm}$. Size of mass and latestage malignancy correlate with hypoxia. ${ }^{10,11}$ Hence, high histopathological grade found in this study was possibly because of chronic hypoxia that occured in the tumor microenvironment which affects the degree of cancer cells differentiation. The parameters associated with breast cancer cell differentiation according to the NGS are the degree of tubular formation and nuclear pleomorphism. ${ }^{11}$

One of the most important elements for mass growth is vascularization. Bigger masses further decrease oxygen diffusion and affect vascular growth. Blood vessels become more irregular and changes occur due to the presence of neovascularisations. As the consequence, there is a fluctuation of oxygen and glucose concentration which ultimately creates a hypoxic, anaerobic environment and aerobic glycolysis. Transient hypoxia occurs due to decrease of local oxygen. If a cell is above its oxygen diffusion limit, chronic hypoxia occurs. ${ }^{12}$ Masses with low differentiation have characteristic of comedo-type necrosis which indicates that the cancerous tissue has necrotic and 
hypoxic area. Therefore, this implies that hypoxia affects the invasive ability of a cancerous cell. ${ }^{13}$

In tubular formation, the presence of polarized and differentiated epithelial cells oriented with the neighboring cells is required. However, hypoxic condition in tumor microenvironment inhibits cancerous cell differentiation and thus those cells lose the ability to form a polarized and organized structure. Rapid growth of cancerous cell causes cancerous and stromal cells far from blood vessels to experience reduced oxygen supply and hence hypoxia. At this rate, those cells shift to glycolytic metabolism which result in acidic microenvironment. Lactate products from the acidic condition can trigger the acceleration of cancerous cell growth. Specifically, cancerous cell in hypoxic environment can promote transformation of normal fibroblast to Cancer Associated Fibroblast (CAF), and after 80 hours of hypoxic tumor microenvironment, CAF can alter the extracellular matrix to become rigid and parallel, as previously known that cancer cells migrate through this parallel matrix. ${ }^{13}$

Moreover, hypoxia increases nuclear pleomorphism ratio which is a special marker for poor cancer cell differentiation where it can lead to fibrosis and form speculation. Specifically, it can promotes transformation of normal fibroblast into CAF and alters extracellular matrix to become stiff and parallel which may be seen as spiculation in mammography. ${ }^{13}$ Hypoxic cell also has decreased expression of differentiation marker including estrogen receptors (ER) in ER positive breast cancers. ${ }^{10,14}$ It was found a decrease in level of ER $\alpha$ protein caused by deregulated adipocytes in hypoxic condition while HIF- $1 \alpha$ gene expression is increased, resulting in growth of tumor and less efficient hormonal therapy. ${ }^{12}$ Jogi A et al (2012) performed human breast cancer cell culture under hypoxic condition and found decreased regulation of ER expression as well as regulation of CK19, and it is evident both in vitro and in vivo that hypoxic condition promotes less differentiated breast cancer phenotype. ${ }^{14}$

NGS is relating the mitotic index parameter with cancerous cell proliferation. This result is similar to what Roychowdhury mentioned as "tumor with $3+3+1$ pattern (tubular formation $<10 \%$, high nuclear pleomorphism, but with low mitotic pattern)". Low mitotic index can be caused by failure of tumor tissue storage post-excision (there is a possibility of exposure to room temperature for quite some time before fixation using formalin was performed), resulting in disappearance of mitotic pattern because those tumor cells have completed their mitotic stages. Hence, for a better accuracy in evaluating tumor cell proliferation index, biomarker Ki67 has been suggested. ${ }^{15}$

The limitations of this study including the small sample size due to some cases having surrounded by soft tissue oedema that blurs the speculation. Some cases already had neoadjuvant chemotherapy. And some cases only had cytological biopsy in the form of fine needle aspiration biopsy (FNAB) instead of tissue pathological result.

\section{Conclusion}

Malignant spiculated masses with histopathological grade I and II have low proliferation index, grade II and III have poor cellular differentiation as well as intermediate to high nuclear pleomorphism. Most spiculated masses found in histopathological grade III.

\section{Conflict of Interest}

The author stated there is no conflict of interest

\section{References}

1. Sun S, Zhang B, Zhao H, Cao X. Association between mammographic features and clinicopathological characeteristics in invasive ductal carcinoma of breast cancer. Molecular and Clinical Oncology 2014;2:623-629.

2. Health ministry of Indonesia. Kementrian kesehatan ajak masyarakat cegah dan kendalikan kanker. http//www.depkes.go.id/article/ view/17020200002/kementerian-hesehatan-ajak-masyarakat-cegahdan-kendalikan-kanker.html. Accesed 02 Februari 2017.

3. Sun Y, Zhao Z, Yang Z, Xu F, Lu H, et al. Risk factors and prevention of breast cancer. International Journal of Biological Sciences 2017;13(11):1387-1397.

4. Liu S, Wu X, Xu W, Lin Q, Liu X, Li Y. Is there a correlation between the presence of a spiculated mass on mammogram and luminal $\mathrm{A}$ subtype breast cancer? Korean Journal of Radiology 2016;17(6):846852.

5. Fischer U, Baum F, Lutfner-Nagel S. Breast cancer: Diagnostic imaging and therapeutic guidance. New York: Thieme; 2018. p.2-21.

6. Lamb P, Perry N, Vinnicombe S, Wells C. Correlation between ultrasound characteristics, mammographic findings and histological grade in patients with invasive ductal carcinoma of the breast. Clinical Radiology 2000(55):40-44.

7. Boisserie-Lacroix M, Hurtevent-Labrot G, Lippa N, Bullier B, Grogan G. Correlation between imaging and prognostic factor: Molecular classification of breast cancer. Diagnostic and Interventional Imaging 2014;95:227-233.

8. Rakha E, Reis-Filho J, Baehner F, Dabbs DJ, Decker T, Eusebi V, et al. Breast cancer prognostic classification in the molecular era: the role of histological grade. Breast Cancer Research 2010;12(4):207.

9. Moiruchi H, Yamaguchi J, Hayashi H, Ohtani H, Shimokawa I, Abiru $\mathrm{H}$, et al. Cancer cell interaction with adipose tissue: Correlation with the finding of spiculation at mammography. Radiology 2016;279(1):56-64.

10. Kim Y, Lin Q, Glazer P, Yun Z. Hypoxic tumor microenvironment and cancer cell differentiation. Curr Mol Med 2009;9(4):425-434.

11. Muz B, Puente P, Zab F, Azab A. The role of hypoxia in cancer progression, angiogenesis, metastasis, and resistance to therapy. Hypoxia (Auckl) 2015;3:83-92

12. Rausch L, Netzer N, Hoegel J, Pramsohler S. The linkage between breast cancer, hypoxia, and adipose tissue. Frontiers in Oncology 2017;2;211.

13. Petrova V, Annicchiarico-Petruzzelli M, Melina G, Amelio I. The hypoxic tumour microenvironment. Oncogenesis 2018;7:10.

14. Jogi A, Vaapil M, Johansson M, Pahlman S. Cancer cell differentiation heterogeneity and aggressive behaviour in solid tumors. Upsala Journal of Medical Sciences 2012;117(2):217-224.

15. Ross J, Hatzis C, Symmans W, Pusztai L, Hortobagyi G. Commercialized multigene predictors of clinical outcome for breast cancer. The Oncologist 2018;13:477-493. 